\title{
GENERAL-WELL-ORDERED SETS
}

\author{
J. L. HICKMAN
}

(Received 10 April 1972)

Communicated by J. N. Crossley

It is of course well known that within the framework of any reasonable set theory whose axioms include that of choice, we can characterize well-orderings in two different ways:

(1) a total order for which every nonempty subset has a minimal element;

(2) a total order in which there are no infinite descending chains.

Now the theory of well-ordered sets and their ordinals that is expounded in various texts takes as its definition characterization (1) above; in this paper we commence an investigation into the corresponding theory that takes characterization (2) as its starting point. Naturally if we are to obtain any differences at all, we must exclude the axiom of choice from our set theory. Thus we state right at the outset that we are working in Zermelo-Fraenkel set theory without choice.

This is not enough, however, for it may turn out that the axiom of choice is not really necessary for the equivalence of (1) and (2), and if this were so, we would still not obtain any deviation from the "classical" theory. Thus the first section of this paper will be devoted to showing that we can in fact have sets that are well-ordered according to (2) but not according to (1). Naturally we have no hope of reversing this situation, for it is a ZF-theorem that any set well-ordered by (1) is also well-ordered by (2).

These sets will be constructed by using Boolean-valued ZF-models. Since we are only concerned with demonstrating that the investigations in this paper are not completely futile, we shall not go into any more detail with this construction than is absolutely necessary, and shall rely very heavily on Rosser (1969). Once the spade-work of the first section has been done, we can start on the pleasant task of discovering just how much of the classical theory can be recaptured, and where the differences lie. It will become apparent that the theory to be developed bears a resemblance to the theory of Crossley's "quasi-well-orderings", as expounded in his book Crossley (1969). Here again, however, there are points at 
which these two theories diverge, perhaps one of the most marked being at the introduction of the operation of exponentiation.

Let us start by giving formally the fundamental definition:

DefinItion 1. Let $(x,<)$ be a (totally) ordered set. $(x,<)$ is said to be general-well-ordered (gwo) if there is no injection $f: \omega \rightarrow x$ such that $f(n+1)$ $<f(n)$ for each $n \in \omega$. The order-type of a gwo set is called a $g$-ordinal.

Since we shall almost always be concerned with total orderings, we shall omit the word total. As inferred above, every well-ordered set is general-wellordered, and hence the theory of $g$-ordinals includes that of ordinals.

1.

As mentioned previously, we shall use Rosser's construction Rosser (1969) of Boolean-valued ZF-models to obtain a gwo set that is not well-ordered. Unexplained notation can be found in Rosser (1969). We shall also use the unary predicates $\mathrm{FN}, \mathrm{MED}, \mathrm{SM} ; \mathrm{FN}(x)$ means that $x$ is finite, $\operatorname{MED}(x)$ means that $x$ is medial (infinite, but Dedekind finite), whilst $\operatorname{SM}(x)$ means that the power-set $P(x)$ is medial. Clearly $\operatorname{SM}(x) \rightarrow \operatorname{MED}(x)$.

We shall construct a ZF-model containing a densely ordered medial set; in fact the set is SM, but this is irrelevant to our main purpose. Now since the underlying set is medial, it is clearly gwo. On the other hand, no medial set can be well-ordered, since otherwise we would have an ordinal incomparable to $\omega$, contradicting the ZF-theorem on the comparability of ordinals. It will be easily seen afterwards that by modifying our construction slightly, we could produce a ZF-model containing a continuously ordered SM set.

If $X$ is a subset of a complete Boolean algebra, for typographical reasons we deviate from Rosser's notation, and use " $\sup X$ ", "inf $X$ " to denote ithe supremum, infimum respectively of $X$.

Definition 2. Let $\eta$ be the set of rational numbers, ordered by magnitude, and let $S$ be the set of all increasing permutations on $\eta$.

As usual, our model $\mathscr{M}$ will be detrmined by the specification of a complete Boolean algebra $\mathscr{A}$, a group $\mathscr{G}$ of $\mathscr{A}$-automorphisms, and a strongly normal filter of subgroups of $\mathscr{G}$.

DeFINITION 3.

(i) Let $\mathscr{A}$ be the Boolean algebra of regular open sets of the Tychonoff space $2^{\omega \times \eta}$; the elements of the usual sub-basis will be denoted by " $B_{m, u}^{i}$ ", $(i, m, u) \in 2 \times \omega \times \eta . \mathscr{A}$ is complete.

(ii) For any $\sigma \in S$, denote by " $\mathscr{G}_{\sigma}$ " the $\mathscr{A}$-automorphism induced by the transformation $B_{m, u}^{i} \rightarrow B_{m, \sigma u}^{i},(i, m, u) \in 2 \times \omega \times \eta$. Define $\mathscr{G}$ to be the group generated by $\left\{g_{\sigma} ; \sigma \in S\right\}$. 
(iii) For any $K \in P(\eta)$, denote by " $\mathscr{G}$ "' that subgroup of $\mathscr{G}$ consisting of those elements of $\mathscr{G}$ that leave $B_{m, u}^{i}$ invariant whenever $u \in K$. Put

$$
\mathscr{F}=\left\{\mathscr{H} \leqq \mathscr{G} ; \exists K\left(K \in P(\eta) \& \mathrm{FN}(K) \& \mathscr{G}^{K} \leqq \mathscr{H}\right)\right\}
$$

$\mathscr{F}$ is a strongly normal filter.

(iv) Define $\mathscr{M}$ by specifying $\mathscr{A}, \mathscr{G}, \mathscr{F}$ as in (i)-(iii) above.

Definition 4. For each $u \in \eta$, define the map $a_{u}: \mathscr{V}_{\omega} \rightarrow \mathscr{A}$ by

$$
a_{u}(x)=\sup \left\{B_{m, u}^{1} \Delta\|x=m\| ; m \in \omega\right\}, \quad x \in \mathscr{V}_{\omega} .
$$

THEOREM 1. The $a_{u}, u \in \eta$, are pairwise distinct $\mathscr{M}$-sets.

PROof. Theorems 6.3, 6.4 of Rosser (1969).

These $a_{u}$ are going to be the elements of our ordered medial set, which we now define.

Definition 5. Define the map $a: \mathscr{V}_{\omega+1} \rightarrow A$ by

$$
a(x)=\sup \left\{\left\|x=a_{u}\right\| ; u \in \eta\right\}, \quad x \in \mathscr{V}_{\omega+1} .
$$

THeOREM 2. $a$ is an $\mathscr{M}$-set, and for each $\mathscr{M}$-set $x$, we have $\mathscr{M} \vDash x \in a$ if and only if $\mathscr{M} \vDash a_{u}=x$ for some $u \in \eta$.

Proof. Extensionality is immediate, and since each $g \in \mathscr{G}$ acts as a permutation of the $a_{u}$, we have $\mathscr{G}_{a}=\mathscr{G} \in \mathscr{F}$ ((3.36) of Rosser (1969). Thus $a$ is an $\mathscr{M}$-set. Clearly $\left\|a_{u} \in a\right\|=1$; hence if $\mathscr{M} \vDash x=a_{u}$, then $\mathscr{M} \vDash x \in a$. A moment's consideration shows that Theorem 3.10 of Rosser (1969) can be applied here, which gives the converse implication.

THEOREM 3. $\mathscr{M} \vDash \operatorname{MED}(a)$.

Proof. Let $f: \omega \rightarrow a$ be an $\mathscr{M}$-function; we shall show that we cannot have $\mathscr{M} \vDash$ In $(f)$, where "In" denotes injectivity. Assume $\mathscr{M} \vDash$ In $(f)$, and let $K \in P(\eta)$ be such that $\mathrm{FN}(K)$ and $\mathscr{G}^{\mathrm{K}} \leqq \mathscr{G}_{f}$. From Theorem 2 it follows that for some $(n, u) \in \omega \times \eta-K$ we have $\mathscr{M} \vDash f(n)=a_{u}$. Let $K=\left\{m_{0}, \cdots, m_{k}\right\}$, with $m_{i}<m_{i+1}$, and assume $m_{j}<u<m_{j+1}$ (if $u<m_{0}$ or $u<m_{k}$, increase $K$ appropriately). Now choose $\sigma \in S$ such that $\sigma(v)=v$ for $v \notin\left(m_{j}, m_{j+1}\right), \sigma(v) \neq v$ for $v \in\left(m_{j}, m_{j+1}\right)$. Then $\sigma \in \mathscr{G}^{K}$, and so we must have $\mathscr{M} \vDash f(n)=a_{\sigma u}$, whence, since $f$ is an $\mathscr{M}$-function, we must have $\mathscr{M} \vDash a_{u}=a_{\sigma u}$. Since $\sigma(u) \neq u$, this contradicts Theorem 1.

Definition 6. Define the map $b: \mathscr{V}_{\omega+3} \rightarrow \mathscr{A}$ by

$$
b(x)=\sup \left\{\left\|x=\left(a_{u}, a_{v}\right)\right\| ; u, v \in \eta, u<v\right\}, \quad x \in \mathscr{V}_{\omega+3} .
$$

THeOREM 4. $b$ is an $\mathscr{M}$-(dense order on $a$ ). 
Proof. Clearly $b$ is extensional, and $\mathscr{G}_{b}=\mathscr{G} \in \mathscr{F}$; hence $b$ is an $\mathscr{M}$-set. As in Theorem 2, we can show that for any $\mathscr{M}$-set $x$ we have $\mathscr{M} \vDash x \in b$ if and only if $\mathscr{M} \mid=x=\left(a_{u}, a_{v}\right)$ for some $u, v \in \eta$ with $u<v$. The rest of the theorem now follows routinely.

We now have an example of an ordered medial set, and hence of a set that is general-well-ordered but not well-ordered. It follows incidentally from what has been proved so far that it is not provable in ZF that every densely ordered set has a subset of type $\eta$.

We now show, via a lemma, that $\mathscr{M} \vDash \operatorname{SM}(a)$.

Lemma 1. Let $c$ be an $\mathscr{M}$-subset of $a$, and let $K=\left\{r, \cdots, r_{k}\right\} \in P(\eta)$ be such that $\mathscr{G}^{K} \leqq \mathscr{G}_{c}$, where $r_{i}<r_{i+1}, i<k$. Assume $\mathscr{M} \vDash \sim \mathrm{FN}(c)$. Then one of the following three cases must hold.

(i) $\mathscr{A} \vDash a_{u} \in c$ for each $u<r_{0}$;

(ii) $\mathscr{M} \vDash a_{u} \in c$ for each $u>r_{k}$;

(iii) $\mathscr{M} \vDash a_{u} \in c$ for each $u \in\left(r_{i}, r_{i+1}\right)$, for some $i<k$.

Proof. By the assumption $\mathscr{M} \vDash \sim F N(c)$, we must have $\mathscr{M} \vDash a_{u} \in \mathcal{c}$ for some $u$ such that

(I) $u<r_{0}$,

(II) $u>r_{k}$,

(III) $u \in\left(r_{i}, r_{i+1}\right)$ for some $i<k$.

As might be expected, we show that (I) $\rightarrow$ (i), (II) $\rightarrow$ (ii), (III) $\rightarrow$ (iii). Since all three cases are analogous, we consider only the last. Choose $v \in\left(r_{i}, r_{i+1}\right)$; it is tedious but routine to construct $\sigma \in S$ such that $g_{\sigma} \in \mathscr{G}^{K}$ and $\sigma(u)=v$. Thus $\mathscr{M} \vDash a_{\nu} \in c$.

\section{THEOREM 5. $\mathscr{M} \mid=\operatorname{SM}(a)$.}

Proof. Assume that we have an $\mathscr{M}$-injection $f: \omega \rightarrow P(a)$, and let $K \in P(\eta)$ be such that $\mathrm{FN}(K)$ and $\mathscr{G}^{K} \leqq \mathscr{G}_{f}$; it is easy to see that we have $\mathscr{G}^{K} \leqq \mathscr{G}_{f(n)}$ for each $n$. It now follows from Lemma 1 that we can assume $\mathrm{FN}(f(n))$ for each $n$. Since $a$ is ordered, however, the assumption $\operatorname{In}(f)$ enables us to construct, by means applicable in ZF and hence in $\mathscr{M}$, an injection $f_{1}: \omega \rightarrow a$, which contradicts Theorem 3.

Suppose now we go back to Definition 1 , replace $\eta$ by the set $\lambda$ of reals ordered by magnitude, and replace $S$ by the corresponding set relative to $\lambda$. By going through precisely the same construction, we would arrive at a ZF-model containing a continuously ordered SM set. 
2.

The results obtained in the last section are encouraging in that they show that an investigation of general-well-order, as distinct from the classical investigations of well-order, is at least not necessarily doomed to futility. Since, however, our main interest lies in the arithmetic of $g$-ordinals, we must first of all satisfy ourselves that certain elementary properties are possessed by $g$-ordinals. We define a map $f: A \rightarrow B$ between two ordered sets to be a similarity if it is orderpreserving, and say that $A, B$ are "similar" $(A \simeq B)$ if there exists a surjective similarity $f: A \rightarrow B$.

LEMMA 2.

(i) For any gwo set $A$ and any similarity $f: A \rightarrow A$, we have $x \leqq f(x)$ for each $x \in A$.

(ii) Let $A, B$ be gwo sets, and let $f, g: A \simeq B$ be surjective similarities (isomorphisms). Then $f=g$.

(iii) Let $A, B$ be gwo sets, with $A$ a segment of $B$, and $A \neq B$. Then $A$ and $B$ are not similar.

Proof. (i) If for some $x^{0} \in A$ we had $f\left(x^{0}\right)<x^{0}$, then $\left(f^{n}\left(x^{0}\right)\right)$ would be an infinite descending chain in $A$.

(ii), (iii) follow immediately from (i).

Lemma 2 (ii) is important because it allows us in certain cases to define the sum of more than a finite number of $g$-ordinals. For the moment, however, we confine ourselves to the finite case and prove closure for addition and multiplication of $g$-ordinals.

THEOREM 6. The class of g-ordinals is closed under finite addition and multiplication of order types.

Proof. The case of addition is trivial, since if the ordered sum of two ordered sets contains an infinite descending chain, then so must one of the components.

Multiplication is also straightforward, for let $(B,<)$ be the ordered product of gwo sets $\left(B^{\prime},<^{\prime}\right)$ and $\left(B^{\prime \prime},<^{\prime \prime}\right)$, and suppose that $f: \omega \rightarrow B$ is such that $f^{\prime \prime} \omega$ has type $\omega^{*}$. Then if $p r_{0}, p r_{1}$ are the projection maps, we have $p r_{1}(f(n+1))$ $\leqq p r_{1}(f(n))$ for each $n$, and so, since $B^{\prime \prime}$ is gwo, there is some $m$ such that $p r_{1}(f(m+n))=p r_{1}(f(m))$ for each $n$. But now $\left(p r_{0}(f(m+n))\right)$ is an infinite descending chain in $B^{\prime}$; contradiction.

At this stage we note a theorem on well-ordered sets that fails in the more general case of gwo sets: it is not necessarily true that if $A$, a gwo set, is similar to a subset of a gwo set $B$, then $A$ is similar to a segment of $B$. For let $D$ be any 
medial set, and put $A=\omega, B=D+\omega$, where "+" denotes ordered union. Then clearly $A$ is similar to a remainder of $B$ but not to any segment of $B$.

THEOREM 7. Let: $\alpha, \beta$ be g-ordinals such that for some order types $\gamma_{0}, \gamma_{1}$ we have $\alpha+\gamma_{0}=\alpha+\gamma_{1}=\beta$. Then $\gamma_{0}, \gamma_{1}$ are g-ordinals, and $\gamma_{0}=\gamma_{1}$.

Proof. Let $A, C_{0}, C_{1}$ be representative sets for $\gamma, \gamma_{0}, \gamma_{1}$ : clearly the $C_{i}$ are gwo, and our hypotheses allow us to assume the existence of an isomorphism $f: A+C_{0} \simeq A+C_{1}$. If either $f(c) \in A$ for some $c \in C_{0}$, or $f(a) \in C_{1}$ for some $a \in A$, then $A$ is similar to a proper segment of itself, contrary to Lemma 2 (ii). Thus $f^{\prime \prime} A=A$, and so $f^{\prime \prime} C_{0}=C_{1}$; hence $\gamma_{0}=\gamma_{1}$.

Definition 7. (i) If $\alpha, \beta$ are $g$-ordinals with $\gamma \neq 0$ a $g$-ordinal such that $\alpha+\gamma=\beta$, then we say that $\alpha$ is less than $\beta$, and write " $\alpha<\beta$ ". (ii) If $\alpha, \beta$ are $g$-ordinals with $\alpha \leqq \beta$, then we denote by " $\beta-\alpha$ " the unique $g$-ordinal $\gamma$ such that $\alpha+\gamma=\beta$.

Now in classical ordinal theory $\omega$ is the least transfinite ordinal, whilst in classical cardinal theory its cardinal has the same property. In non-classical cardinal theory this minimal property is lost; when we consider general-wellorder, however, it is partially regained:

THEOREM 8. Let $\alpha$ be a g-ordinal such that $n \leqq \alpha$ for each $n \in \omega$. Then $\omega \leqq \alpha$.

Proof. Let $A$ be a representative set for $\alpha$. Lemma 2 (ii) tells us that there is a unique similarity $f_{n}: n \rightarrow A$ such that $f^{\prime \prime} n$ is a segment of $A$. Define $f: \omega \rightarrow A$ by $f(n)=f_{n+1}(n)$. Clearly $f$ is a similarity, and $f_{n}^{\prime \prime} \omega$ is a segment of $A$. This proves our theorem.

We now return to the result mentioned previously, namely that in some cases it is possible to define generalized addition of $g$-ordinals.

THEOREM 9. Let $\left\{A_{x}\right\}_{x \in X}$ be an indexed set of gwo sets, where the indexing set $X$ is also gwo. Then the ordered union $\Sigma\left\{A_{x} ; x \in X\right\}=A$ is gwo.

Proof. Suppose that $f: \omega \rightarrow A$ is an infinite descending chain in $A$. We may assume that the $A_{x}$ are pairwise disjoint, and so for each $a \in A$ there exists a unique $s(a) \in X$ such that $a \in A_{s(a)}$. Using the fact that each $A_{x}$ is gwo, we may define a function

$$
\begin{aligned}
& g: \omega \rightarrow X \text { recursively by } g(0)=s(f(0)), \\
& g(n+1)=s\left(f\left(\min \left\{k ; n<k \& f(k) \notin A_{g(n)}\right\}\right)\right) .
\end{aligned}
$$

It is easy to see that $g(n+1)<g(n)$ for each $n$, which is a contradiction. 
Using Lemma 2 (ii), we can legitimately define the sum of an ordered indexed set of $g$-ordinals; by Theorem 9 , this sum will be a $g$-ordinal whenever the indexing set is gwo.

Theorem 9 allows us to demonstrate the failure of another well-known result on well-ordered sets when extended to general-well-order; namely that if two well-ordered sets are each similar to a subset of the other, then they are similar.

To refute this for gwo sets, we consider the sequences $\left(A_{n}\right)_{n \in \omega},\left(B_{n}\right)_{n \in \omega}$ of gwo sets defined as follows. Let $D$ be a fixed dense gwo set, and put

$A_{2 n}=B_{2 n+1}=\omega, B_{2 n}=A_{2 n+1}=D, A=\Sigma\left\{A_{n} ; n \in \omega\right\}, B=\Sigma\left\{B_{n} ; n \in \omega\right\}$.

Then it is easy to see that $A$ and $B$ are each similar to a subset of the other, but are not similar.

Unlike the class of ordinals, we do not have total comparability of $g$-ordinals. We do have some compensation in this respect, however, in that the ordering of $g$-ordinals is what Crossley in Crossley (1969) calls a "tree-ordering".

Lemma 3. Let $\alpha, \beta, \gamma$ be g-ordinals such that $\alpha, \beta \leqq \gamma$. Then either $\alpha \leqq \beta$ or $\beta \leqq \alpha$.

Proof. Let $A, B, C$ be representative sets for $\alpha, \beta, \gamma$; then by assumption there exist gwo sets $D, E$, and an isomorphism $f: B+E \simeq A+D$. Now either $f^{\prime \prime} B$ is a segment of $A$, in which case we are through, or there is some $x \in B$ such that $f(x) \in D$. In this case, however, we have $f^{-1} A$ being a segment of $B$.

The fact that this ordering is a tree-ordering, together with the fact that it is consistent to assume the existence of incomparable $g$-ordinals, tells us that a third result on ordinals fails for $g$-ordinals: given a set of $g$-ordinals, there is not necessarily a $g$-ordinal that is an upper bound for that set.

The preceding lemma provides us with a quick proof of the "Directed Refinement Theorem" for $g$-ordinals.

THEOREM 10. Let $\alpha, \beta, \gamma, \delta$ be g -ordinals such that $\alpha+\gamma=\beta+\delta$. Then there exists a g-ordinal $\xi$ such that either $\alpha+\xi=\beta$ and $\xi+\delta=\gamma$, or else $\beta+\xi=\alpha$ and $\xi+\gamma=\delta$.

Proof. We have $\alpha, \beta \leqq \alpha+\gamma$; hence by Lemma 3 there exists a $g$-ordinal $\xi$ such that either

(i) $\alpha+\xi=\beta$, or

(ii) $\beta+\xi=\alpha$.

(i) We have $\alpha+\gamma=\beta+\delta=(\alpha+\xi)+\delta=\alpha+(\xi+\delta)$, and so by Theorem 7 we have $\gamma=\xi+\delta$.

(ii) Analogous.

We conclude this section by proving a cancellation law for multiplication of $g$-ordinals. 
THEOREM 11. Let $\alpha, \beta, \gamma$ be g-ordinals with $\alpha \neq 0$ and $\alpha \beta=\alpha \gamma$. Then $\beta=\gamma$.

Proof. Let $A, B, C$ be representative sets for $\alpha, \beta$, $\gamma$, with $f: A \times B \simeq A \times C$ the isomorphism. For $b \in B$, put $S(b)=\{x \in B ; x \leqq b\}, S^{\prime}(b)=S(b)-\{b\}$, and for $c \in C$ define $S(c), S^{\prime}(c)$ similarly. Take $b \in B$, and consider the segment $A \times S(b)$ of $A \times B$ : then $D=f^{\prime \prime}(A \times S(b))$ is a segment of $A \times C$. We shall show that there is a unique segment $R$ of $C$, a unique proper segment $E$ of $A$, and a unique $c \in C$ such that $D=(A \times R)+(E \times\{c\})$.

We define $R$ by $R=\{x \in C ; \forall a \in A((a, x) \in D)\}$; since $D$ is a segment of $A \times C$, it follows that $R$ is a segment of $C$.

By definition of $R$, we have a proper segment $E$ of $A$ and an element $c$ of $C$ such that $D=(A \times R)+(E \times\{c\})$; the uniqueness of $R$ and $E$, and thereby of $c$, follows from the fact that we are dealing with segments of given gwo sets, and so by Lemma 3 have comparability.

Next we show that $R$ has a last element, $R=S\left(c^{\prime}\right)$ for some $c^{\prime} \in C$. Suppose not; we note that $A \times S(b)=A \times S^{\prime}(b)+(A \times\{b\})$, and so since $E \neq A$, we must have $f(a, b) \in A \times R$ for some $a \in A$. Put $f(a, b)=\left(a^{\prime}, r\right) \in A \times R$, and choose any $r^{\prime} \in R$ with $r<r^{\prime}$; this is possible as we are assuming that $R$ has no last element. Then $f^{-1}\left(A \times\left\{r^{\prime}\right\}\right)$ would be a proper subset of $A \times\{b\}$, which is a contradiction. Hence we must have $R=S\left(c^{\prime}\right)$ for some $c^{\prime} \in C$.

We now show that $E=\phi$. Suppose not; then since $E$ is a proper segment of $A$, we see that $f^{-1}\left(A \times\left\{c^{\prime}\right\}\right) \cap A \times\{b\}$ is neither $A \times\{b\}$ nor $\phi$. This means that for some $a, a^{\prime} \in A, b^{\prime} \in S^{\prime}(b)$, we must have $\left(a^{\prime}, b^{\prime}\right),(a, b) \in f^{-1}\left(A \times\left\{c^{\prime}\right\}\right)$, and it follows from this that $S^{\prime}(b)$ has a last element, say $b_{1}$; that is, $S^{\prime}(b)=S\left(b_{1}\right)$.

We now consider $A \times S\left(b_{1}\right)$, and see that $f^{\prime \prime}\left(A \times S\left(b_{1}\right)\right)=A \times S^{\prime}\left(c^{\prime}\right)+E^{\prime} \times\left\{c^{\prime}\right\}$ for some unique nonzero proper segment $E^{\prime}$ of $A$. Since $c>c^{\prime}$, it follows that we can give an inductive definition of an infinite descending chain $c>c^{\prime}>c^{\prime \prime}>\cdots$ in $C$, contradicting the fact that $C$ is gwo. Thus $E=\phi$. We have thus far shown the following. Given any $b \in B$, there exists a unique $c_{b} \in C$ such that $f^{\prime \prime}(A \times S(b))$ $=A \times S\left(c_{b}\right)$. We now do the obvious thing and define a function $g: B \rightarrow C$ by $g(b)=c_{b}$; it is routine to show that for any $b, b^{\prime} \in B$, if $b \leqq b^{\prime}$, then $g(b) \leqq g\left(b^{\prime}\right)$.

By repeating the whole process, only this time starting with $f^{-1}: A \times C$ $\simeq A \times B$, we can define a function $h: C \rightarrow B$, and it is straightforward to check that $h g=1_{B}, g h=1_{C}$. Thus $g$ is an isomorphism, and so $\beta=\gamma$.

We note that this cancellation law is necessarily one-sided, since we do not have right-hand cancellation even in the classical case; the same remark, of course, applies also to additive cancellation.

We can essentially distinguish four branches of this field of study. Firstly, on the side of recursion theory, we have the theory of isols, as expounded by Dekker and Myhill. Secondly, still in recursion theory, we have the theory of losols, and more generally quords, as given by Crossley; this, generally, con- 
stitutes the order analogue of isols. Crossing from recursion theory to set theory, we arrive at the theory of medial cardinals, which corresponds to the theory of isols. Finally, if we try to find a set-theoretic analogoue to quords, we obtain $g$-ordinals (which, I suppose, should be called "geords"). In three of these cases - isols, quards, $g$-ordinals - we have a multiplicative cancellation law. Only in the case of medial cardinals is such a law not apparently forthcoming, and though I know of no counterexample to it, I suspect that in this case cancellation does not necessarily hold.

\section{3.}

Theorem 9 in the preceding section tells us that if $\left\{A_{x}\right\}_{x \in X}$ is an indexed set of gwo sets with the indexing set $X$ also being gwo, then the ordered union is !gwo. It is not immediately clear, however, that if $X$ is ordered, and the ordered union $A=\Sigma\left\{A_{x} ; x \in X\right\}$ is gwo, then $X$ must be gwo; possibly the reason why this is not clear is that it is false; and the purpose of this section is to demonstrate this.

Specifically, we shall construct a Boolean ZF-model containing a gwo set $A=\Sigma\left\{A_{x} ; x \in X\right\}$ such that although each $A_{x}$ is (necessarily) gwo, the ordered indexing set $X$ has order type $\omega^{*}$.

Definition 8. For each $n \in H, N$ being the set of natural numbers, let $R_{n}$ be the real open interval $(n, n+1)$, and put $R=\Sigma\left\{R_{n} ; n \in H\right\}, I=H \times R$. Let $A$ be the (complete) Boolean algebra of regular open sets of the Tychonoff space $2^{I}$.

The elements of the usual subbasis will be denoted by " $B_{n r}^{i}$ ": $B_{n, r}^{i}$ $=\left\{f \in 2^{I} ; f(n, r)=i\right\}$. We note that each $R_{n}$ possesses a natural order of type $\lambda$; we shall refer to each such ordering as " $<$ ". However, we do not assign an ordering to $R$ until we assign one to $N$ (since this will not be the natural ordering, we have refrained from our usual practice of denoting this set by " $\omega$ ").

Definition 9. We define a set $Q$ or permutations on $R$ as follows: $q \in Q$ if and only if for each $n, q \mid R_{n}$ is an automorphism of $R_{n}$.

Since each permutation on $I$ induces a permutation on the subbasis, which in turn produces an $\mathscr{A}$-automorphism, we can define our group $\mathscr{G}$ of $\mathscr{A}$-automorphisms:

Definition 10. For each $q \in Q$, let $g_{q}$ be the $\mathscr{A}$-automorphism induced by the subbasis transformation $B_{n, r}^{i} \rightarrow B_{n, q(r)}^{i},(i, n, r) \in 2 \times I$. Now let $\mathscr{G}$ be the group generated by the set $\left\{g_{q} ; q \in Q\right\}$ of $\mathscr{A}$-automorphisms.

Finally, we define our filter $\mathscr{F}$ according to the usual pattern.

Definition 11. Let $J$ be any subset of $R$, and define $\mathscr{G}^{J}$ to be that subgroup of $\mathscr{G}$ consisting of those $g \in \mathscr{G}$ that leave $B_{n, r}^{i}$ invariant whenever $r \in J$. Now put 


$$
\mathscr{F}=\left\{\mathscr{H} \leqq \mathscr{G} ; \exists J \in P(R)\left(\mathrm{FN}(J) \& \mathscr{G}^{J} \leqq \mathscr{H}\right\} .\right.
$$

A routine check shows that $\mathscr{F}$ is a strongly normal filter. Hence

Definition 12 . Let $\mathscr{M}$ be the ZF-model determined by specifying $\mathscr{A}, \mathscr{G}, \mathscr{F}$ as above.

Definition 13. For each $r \in R$, define the map $a_{r}: \mathscr{V}_{\omega} \rightarrow \mathscr{A}$ by

$$
a_{r}(x)=\sup \left\{B_{n}{ }^{1} \Lambda\|x=\hat{r}\| ; n \in H\right\}, \quad x \in \mathscr{V}_{\omega} .
$$

THEOREM 12. The $a_{r}$ are pairwise distinct $\mathscr{M}$-sets.

Proof. Routine; see Theorems 6.3, 6.4 of Rosser (1969).

Definition 14. For each $n \in H$, define the map $A_{n}: \mathscr{V}_{\omega+1} \rightarrow A$ by

$$
A_{n}(x)=\sup \left\{\left\|x=a_{r}\right\| ; r \in R_{n}\right\}, \quad x \in \mathscr{V}_{\omega+1} .
$$

THEOREM 13. Each $A_{n}$ is an $\mathscr{M}$-set, and has the property that for any $\mathscr{M}$-set $x, \mathscr{M} \vDash x \in A_{n}$ if and only if $\mathscr{M} \vDash x=a_{r}$ for some $r \in R_{n}$.

ProOF. $A_{n}$ is clearly extensional, and since each $g \in \mathscr{G}$ acts as a permutation on $R_{n}$, we have $\mathscr{G}_{A_{a}}=\mathscr{G} \in \mathscr{F}$; thus $A_{n}$ is an $\mathscr{M}$-set.

The remainder of the proof follows the corresponding proof in section 1.

Definition 15. Define the function $X: \mathscr{V}_{\omega+2} \rightarrow \mathscr{A}$ by

$$
X(x)=\sup \left\{\left\|x=A_{n}\right\| ; n \in H\right\}, \quad x \in \mathscr{V}_{\omega+2} .
$$

THeOREM 14. $X$ is an $\mathscr{M}$-set, and for any $\mathscr{M}$-set $x, \mathscr{M} \vDash x \in X$ if and only if $\mathscr{M} \vDash x=A_{n}$ for some $n \in H$.

Proof. As usual.

Definition 16. $A=\mathscr{Q} X$.

THEOREM 15. $A$ is an $\mathscr{M}$-set, and $\mathscr{M} \vDash \operatorname{MED}(A)$.

Proof. The fact that $A$ is an $\mathscr{M}$-set follows from the fact that $\mathscr{M}$ is a ZFmodel. Clearly $\mathscr{M} \vDash \sim \mathrm{FN}(A)$, so suppose that there is an $\mathscr{M}$-injection $f: H \rightarrow A$, and let $J \in P(R)$ be such that $\mathrm{FN}(J)$ and $\mathscr{G}^{J} \in \mathscr{G}_{f}$. Thus we may assume that $\mathscr{M} \vDash f(n)=a_{r}$ for some $(n, r) \in N \times(R-J)$ : we note at this stage that the $A_{n}$ are pairwise disjoint. However, there clearly exists $g \in \mathscr{G}^{J}$ with $g\left(a_{r}\right)=a_{s}$, with $r \neq s$. This gives the desired contradiction.

THEOREM 16. For each $n, \mathscr{M} \vDash \operatorname{MED}\left(A_{n}\right)$.

ProOF. $\mathscr{M} \vDash \sim \mathrm{FN}\left(A_{n}\right) \& A_{n} \in P(A)$.

THEOREM 17. $\mathscr{M} \vDash N \simeq X$. 
Proof. We need to construct an $\mathscr{M}$-bijection $f: N \simeq X$. Thus we define a $\operatorname{map} f: \mathscr{V}_{\omega+4} \rightarrow \mathscr{A}$ by

$$
f(x)=\sup \left\{\left\|x=\left(\hat{n}, A_{n}\right)\right\| ; n \in H\right\}, \quad x \in \mathscr{V}_{\omega+4} .
$$

Clearly $f$ is extensional, and $\mathscr{G}_{f}=\mathscr{G}$; hence $f$ is an $\mathscr{M}$-set. We show in the usual way that for any $\mathscr{M}$-set $x, \mathscr{M} \vDash x \in f$ if and only if $\mathscr{M} \vDash x=\left(\hat{n}, A_{n}\right)$ for some $n \in N$, from which the desired result follows.

Definition 17. For each $n \in H$, define a map $O_{n}: \mathscr{V}_{\omega+2} \rightarrow \mathscr{A}$ by

$$
O_{n}(x)=\sup \left\{\left\|x=\left(a_{r}, a_{s}\right)\right\| ; r, s \in R_{n} \& r<s\right\}, \quad x \in \mathscr{V}_{\omega+2} .
$$

THEOREM 18. Each $O_{n}$ is an $\mathscr{M}$-set, and for any $\mathscr{M}$-set $x, \mathscr{M} \vDash x \in O_{n}$ if and only if $\mathscr{M} \vDash x=\left(a_{r}, a_{s}\right)$ for some $r, s \in R_{n}$ with $r<s$.

PRoof. Most of this is just routine; we merely observe that all $q \in Q$ are by definition order-preserving on each $R_{n}$, so that $\mathscr{G}_{n}=\mathscr{G}$.

THEOREM 19. $\mathscr{G} \mathrm{F}\left(O_{n}\right.$ is a linear order on $\left.A_{n}\right)$.

Proof. Immediate from Theorem 18.

We have thus constructed within $\mathscr{M}$ an indexed set $\left\{A_{n}\right\}_{n \in X}$ of gwo sets, in which the indexing set $X$ is countably infinite. Thus we can induce upon $X$ an order of type $\omega^{*}$, after which the union $A=\Sigma\left\{A_{n} ; n \in X\right\}$ becomes an ordered set. Since, however, $A$ is medial, it follows that $A$ is gwo. This gives us our result.

4.

The Hamilton-Nerode theorem on losols states that if $\tau$ is any countable order type then there are $2^{\omega_{0}}$ losols of classical order type $\tau$ that are pairwise incomparable under the natural losolic ordering (see p. 178 of Crossley (1969)).

This section is devoted to a slightly analogous result for $g$-ordinals. Let $\alpha$ be any dense $g$-ordinal of cardinality $\nabla$, and let $x$ be any fixed aleph. We will show that there exists a "set" $S$ of pairwise incomparable $g$-ordinals such that $S$ has cardinality $2^{x}$, and any $\beta \in S$ has cardinality $\kappa(\nabla+1)$.

We proceed as follows. We let $\left(x_{\eta}\right)$ be a transfinite sequence of length $\kappa$, and we split $\left(x_{\eta}\right)$ into two subsequences, $\left(y_{\eta}\right)$ and $\left(z_{\eta}\right)$, each of length $\kappa$. This is possible since, $\kappa$ being an aleph, we have $2 \kappa=\kappa$.

Clearly $\alpha$ and $\kappa$ are incomparable. We define a set $T$ of $g$-ordinals as follows. In $\left(x_{\eta}\right)$ we replace each $x_{\eta}$ by either $\alpha$ or $\kappa$; clearly there are $2^{*}$ such sequences. Since generalized addition of $g$-ordinals is defined, we can form the ordered sums; the resulting $g$-ordinals will be the members of $T$.

We now show that the members of this set $T$ are pairwise incomparable; to this end, we let $\beta, \gamma \in T$ be defined by the sequences $\left(\beta_{\eta}\right),\left(\gamma_{\eta}\right)$, and assume $\beta \neq \gamma$. Then there is a least $\lambda<\kappa$ such that $\beta_{\lambda} \neq \gamma_{\lambda}$. 
Thus we have $\beta_{\eta}=\gamma_{\eta}$ for $\eta<\lambda$, and we put

$$
\delta=\sum_{\eta<\lambda} \beta_{\eta}=\sum_{\eta<\lambda} \gamma_{\eta}
$$

$\delta$ is well-defined by generalized addition of $g$-ordinals. Clearly $\delta<\beta, \gamma$, and hence $\beta-\delta, \gamma-\delta$ are also well-defined. Now we have either $\beta_{\lambda}=\alpha$ and $\gamma_{\lambda}=\kappa$, or vice versa; by symmetry we may assume the former. Hence for some $g$-ordinals $\psi, \zeta$, we have $\beta=\delta+\alpha+\psi, \gamma=\delta+\kappa+\zeta$.

It is now clear that $\beta$ and $\gamma$ are incomparable. For suppose that $\beta+\xi=\gamma$; then $\delta+\alpha+\psi+\xi=\delta+\kappa+\xi$, whence by cancellation we obtain $\alpha+\psi+\xi$ $=\kappa+\xi$, which is obviously impossible. Similarly if $\gamma \leqq \beta$.

Thus we have produced a set $T$ of pairwise incomparable $g$-ordinals such that $T$ has cardinality $2^{x}$. However, not all elements of $T$ have the same cardinality. In order to achieve this last condition, we define the subset $S$ of $T$ to consist of those $g$-ordinals that are determined by the following property: $y_{0}=\kappa$, and $\gamma_{\eta}=\alpha$ for $0<\eta<\kappa$. Since no restriction has been placed on the subsequence $\left(z_{\eta}\right)$, which is of length $\kappa$, it is clear that $S$ has cardinality $2^{x}$. We leave it to the traditional "interested reader" to show that each $g$-ordinal in $S$ has cardinality $\kappa(\nabla+1)$.

\section{5.}

In this final section we define a partial binary operation of exponentiation on $g$-ordinals, and show that the class of $g$-ordinals is closed under this partial operator. This is in contrast to the case of quords, where it has been shown that there is a quord $q$ such that $2^{q}$ is not a quord.

Definition 18. Let $\alpha$ be any $g$-ordinal such that $\alpha \geqq 1$, and let $\beta$ be an arbitrary $g$-ordinal. We define $\alpha^{\beta}$ as follows. Let $A, B$ be representative sets for $\alpha, \beta$ respectively, and let $a$ be the first element of $A$. Define $E \in P\left(A^{B}\right)$ by

$$
E=\left\{f \in A^{B} ; \mathrm{FN}(\{x \in B ; f(x) \neq a\})\right\} .
$$

We order $E$ as follows. For $f, g$ with $f \neq g$, let $z=z_{f, g}=\max \{\in B ; f(x) \neq g(x)\}$. Now set $f<g$ if and only if $f(z)<g(z)$.

It is routine to show that $<$ linearly orders $E$, and that the order type of $(E,<)$ is independent of the choice of $A$ and $B$. Thus we define $\alpha^{\beta}$ to be the order type of $(E,<)$.

It is perhaps in order to make two comments on this definition. Firstly, when $\alpha$ and $\beta$ are ordinals, this is just the standard set-theoretic definition of ordinal exponentiation. Secondly, when we are dealing with $g$-ordinals, the condition " $\alpha \geqq 1$ " is not equivalent to the condition " $\alpha \neq 0$ ": any dense $g$-ordinal is incomparable with 1 .

THEOREM 20. Let $\alpha, \beta$ be g-ordinals, with $\alpha \geqq 1$. Then $\alpha^{\beta}$ is a g-ordinal. 
Proof. Given $A, B, E$, as in Definition 18, assume that $E$ is not gwo; thus there exists an infinite descending chain $f: \omega \rightarrow E$. Let $z_{0}, \cdots, z_{n}$ be those $x \in B$ for which $f(0)(x) \neq a$, and for each $i, j$, with $i<j$, define $y_{i j} \in B$ by

$$
y_{i j}=\max \{x \in B ; f(i)(x) \neq f(j)(x)\} .
$$

We first observe that for each $i$ we have $y_{01}=z_{j}$ for some $j \leqq n$, for otherwise we would have $f(0) \leqq f(i)$ for some $i$. Thus for some $j \leqq n$, we have $y_{0 i}=z_{j}$ for an infinite number of $i$; let $\bar{z}_{0}$ be the greatest such $z_{j}$, and define $f_{0}: \omega \rightarrow E$ by $f_{0}(0)$ $=f\left(\min \left\{i ; y_{0 i}=\bar{z}_{0}\right\}\right), f_{0}(n+1)=f\left(\min \left\{i ; y_{0 i}=\bar{z}_{0} \& f(i) \notin\left\{f_{0}(j)\right\}_{j \leqq n}\right\}\right)$. Clearly $f_{0}$, being a subsequence of $f$, is an infinite descending chain in $E$. We now define $y_{i j}^{0}, i<j$, by $y_{i j}^{0}=\max \left\{x \in B ; f_{0}(i)(x) \neq f_{0}(j)(x)\right\}$, and we see that $y_{1 j}^{0} \leqq \bar{z}_{0}$ for each $j$. For suppose $j^{\prime}$ to be such that $\bar{z}_{0}<y_{1 j^{\prime}}^{0}$.

Since $\bar{z}_{0}=y_{01}^{0}=y_{0 j^{\prime}}^{0}$ (by definition of $\left.f_{0}\right)$, we deduce (by definition of $\bar{z}_{0}$ ) that $f_{0}(1)\left(y_{1 j^{\prime}}^{0}\right)=f_{0}\left(j^{\prime}\right)\left(y_{1 j^{\prime}}^{0}\right)$, contradicting the definition of ${ }_{1 j^{\prime}}^{0}$. Thus we must have $y_{1 j}^{0} \leqq \bar{z}_{0}$ for each $j$.

Reasoning as before, we can infer the existence of a non-empty FN subset $C$ of $B$ such that for each $z \in C$ we have $y_{1 j}=z$ for an infinite number of $j$ : we put $\bar{z}_{1}=\max C$, and we know that $\bar{z}_{1} \leqq \bar{z}_{0}$.

We can now define $f_{1}: \omega \rightarrow E$, and repeat the process ad nauseum, thereby obtaining a nonascending sequence $\left(\bar{z}_{i}\right)$ in $B$; since $B$ is gwo, this sequence has a minimal element, $\bar{z}_{k}=\bar{z}$; the corresponding $f_{k}: \omega \rightarrow E$ is descending and has the property that for all $i, j$, with $i \neq j, \max \left\{z \in B ; f_{k}(i)(z) \neq f_{k}(j)(z)\right\}=\bar{z}$. Thus $\left(f_{k}(i)(\bar{z})\right)$ is a descending chain in $A$; contradiction. Thus $E$ must be gwo.

As yet $I$ have not investigated the arithmetical properties of exponentiation of $g$-ordinals.

We conclude by giving a possible generalization of the concept of general-well-order; to lead into this, we give an alternative characterization of generalwell-order itself.

THEOREM 21. Let $(A,<)$ be an ordered set. Then $A$ is gwo if and only if every non-empty countable subset of $A$ has a first element.

Proof. In one direction this is trivial, since if $A$ has an infinite descending chain $f: \omega \rightarrow A$, then $f^{\prime \prime} \omega$ is non-empty, countable, with no first element. On the other hand if $B$ is a non-empty countable subset of $A$ without first element, then it is straightforward to produce a bijection $f: \omega \rightarrow B$ such that $f(n+1)<f(n)$ for each $n$.

This result suggests the following definition.

Definition 19. Let $\kappa$ be any cardinal. An ordered set $(A,<)$ is said to be $\kappa$-well-ordered if every subset of $A$ of cardinality $\leqq \kappa$ has a first element, and at least one subset of $A$ has cardinality $\kappa$. 
Theorem 21 makes it clear that $\kappa$-well-order is a generalization of generalwell-order; however, the parallel development of the theory of $\kappa$-well-ordered sets seems fraught with so many difficulties as to make one reluctant to pursue it. In this vein I remark without proof that if we modify Definition 19 by substituting

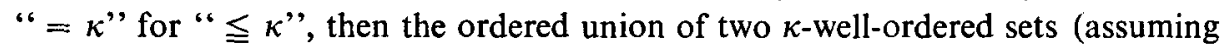
such to exist) is necessarily $\kappa$-well-ordered if and only if $\kappa=\omega_{0}$.

I would like to thank the referee for his stirring comments on the unrevised version of this paper. To him is due the brief proof of Theorem 7, and a considerable simplification of the construction of the Boolean model in section 3 .

The investigations contained in this paper were commenced whilst the author was a Research Assistant at Flinders University, and continued during a Postdoctoral Fellowship at the Australian National University.

\section{References}

J. N. Crossley (1969), Constructive order types (Studies in Logic, North Holland 1969).

J. C. E. Dekker and J. Myhill (1960), 'Recursive equivalence types', Univ. California Publs. Math. n. s. 3, 67-214.

J. L. Hickman (1971), 'Some definitions of finitiness', Bull. Austral. Math. Soc. 5, 321-330.

J. L. Hickman (1972), 'Some definitions of finitiness: Corrigenda', Bull. Austral. Math. Soc. 6, 319.

J. Barkely Rosser (1969), Simplified independence proofs (Academic Press, 1969).

Department of Mathematics

Institute of Advanced Studies

Australian National University

Canberra, ACT 